\title{
A Case of Lung Adenocarcinoma Presenting as Miliary
}

\section{Appearance}

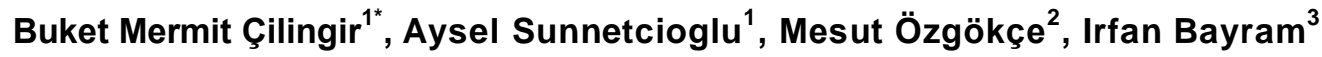 \\ ${ }^{1}$ Department of Pulmonology, Van Yüzüncü Yal University, Van, Turkey \\ ${ }^{2}$ Department of Radiology, V an Yüzüncü Yal University, Van, Turkey \\ ${ }^{3}$ Department of Pathology, V an Yüzüncü Yal University, Van, Turkey
}

\begin{abstract}
Multiple benign conditions may be the cause of miliary nodules, but the most common cause is metastatic disease. Even so miliary metastasis of lung cancer is rarely seen. The most common lung cancer with a miliary distribution is adenocarcinoma. It is often difficult to diagnose adenocarcinomas with a progressive course. Anamnesis, physical examination, imaging methods can give an idea but diagnosis should be confirmed histopathologically. As in our case, tissue biopsy should be performed for diagnosis. We aimed to present this case because we performed a tru cut biopsy which is rarely used for histopathologic diagnosis and the miliary nodules in the lung of adenocarcinoma is a rare condition.
\end{abstract}

Key Words: Lung, Adenocarcinoma, Miliary pattern

\section{Introduction}

The differential diagnoses of miliary patern comprise miliary tuberculosis, fungal inections (cryptococcosis, histoplasmosis, coccidiodomycosis, blastomycosis), sarcoidosis, coal miners pneumoconiosis, silicosis, hemosiderosis, fibrosing alveolitis, acute extrinsic allergic alveolitis, pulmonary eosinophilic syndrome, pulmonary alveolar proteinosis, and hematogenous distribution metastases from the primary cancers of the thyroid, kidney, throphoblasts and some sarcomas $(1,2)$.

The incidence of lung cancer is high in males and peaks between age 55 and 65 years. The miliary pattern in chest radiography is very rare in patients with primary lung cancer $(3,4,5)$. We wanted to present our case becouse of this rare condition.

\section{Case Report}

A 57-year-old man presented with a history of fever, cough, nausea and vomiting lasting for 15 days. He had cough associated with mucoid expectoration, which was non-blood tinged. He was not a smoker or an alcoholic. He was not any knowledge disease. There was no family history of tuberculosis or close contact with tuberculosis. On examination, the patient fewer was $38{ }^{\circ} \mathrm{C}$, a pulse of 86 beats per minute and blood pressure of 120/80 $\mathrm{mmHg}$. General physical examination did not reveal pallor, clubbing, cyanosis, edema, or lymphadenopathy. Hemogram revealed a total count of $10,700 / \mathrm{mm} 3$. The erythrocyte sedimentation rate (ESR) was 32 $\mathrm{mm} / \mathrm{h}$. Sputum acid fast bacilli (AFB) (3 samples) were negative. A Gram stain showed plenty of epithelial cells, Gram-positive cocci, and gramnegative bacilli. Liver function and renal function tests were within normal limits. The Mantoux test was negative. Human immunodeficiency virus (HIV) was nonreactive. Chest X-ray showed reticulonoduler patern and miliary nodules (Figure 1). Thoracic computed tomography (CT) revealed a small, mildly enhancing, nodular lesion containing central density involving the posterior basal segment of the left lower lobe with a few enlarged pretracheal, retrocaval, aortopulmonary, and right hilar lymph nodes (Figure 2).

Broncoscopy was performed to the patient but all results were negative. Because of diffuse involvement on thorax CT, conventional 18-gauge Tru-Cut needle biopsy was planned. A $1.5 \mathrm{~cm}$ linear segment of parenchymal biopsy was obtained from the superior segment of the lower lobe of the left lung. Postoperative complication wasn't seen in the patient.

Pathology preparations were evaluated as; primary lung adenocarcinoma with multiple foci suggestive of a metastatic adenocarcinoma (Figure 3). Immunohistochemical Napsin $\mathrm{A}(+)$ supporting the primary focus of cancer is the lung (Figure 4). Adenocarcinoma metastasis in lymph node biopsy 


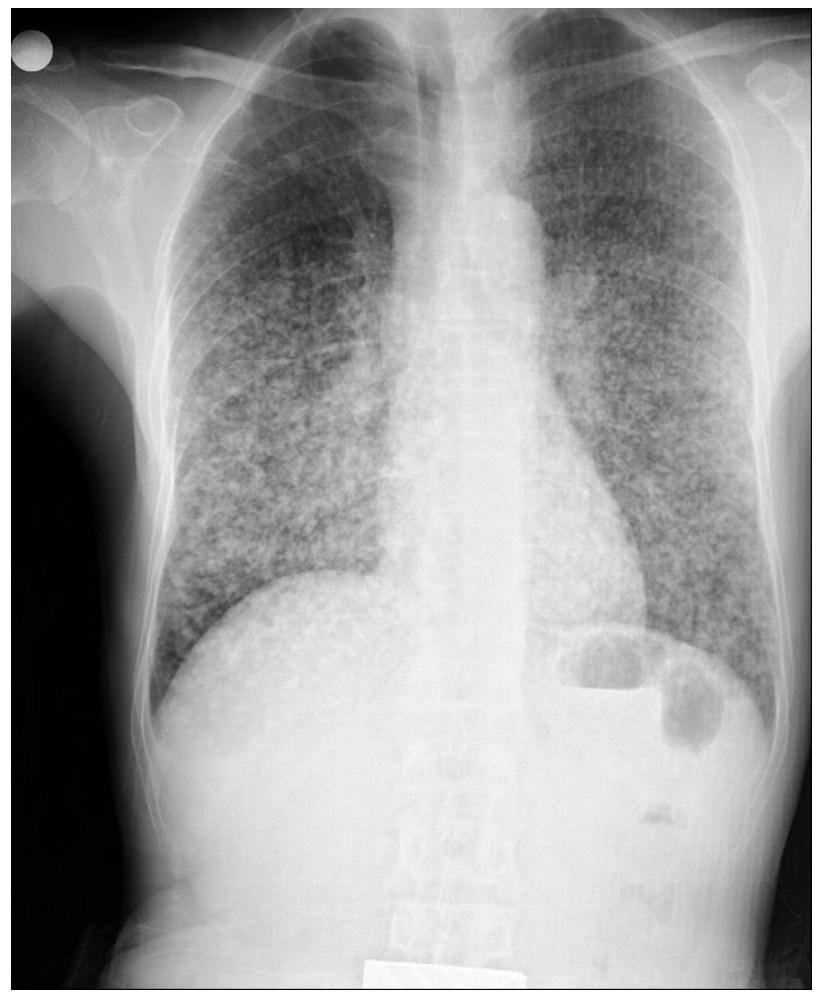

Fig. 1. Reticulonoduler pattern and miliary nodules

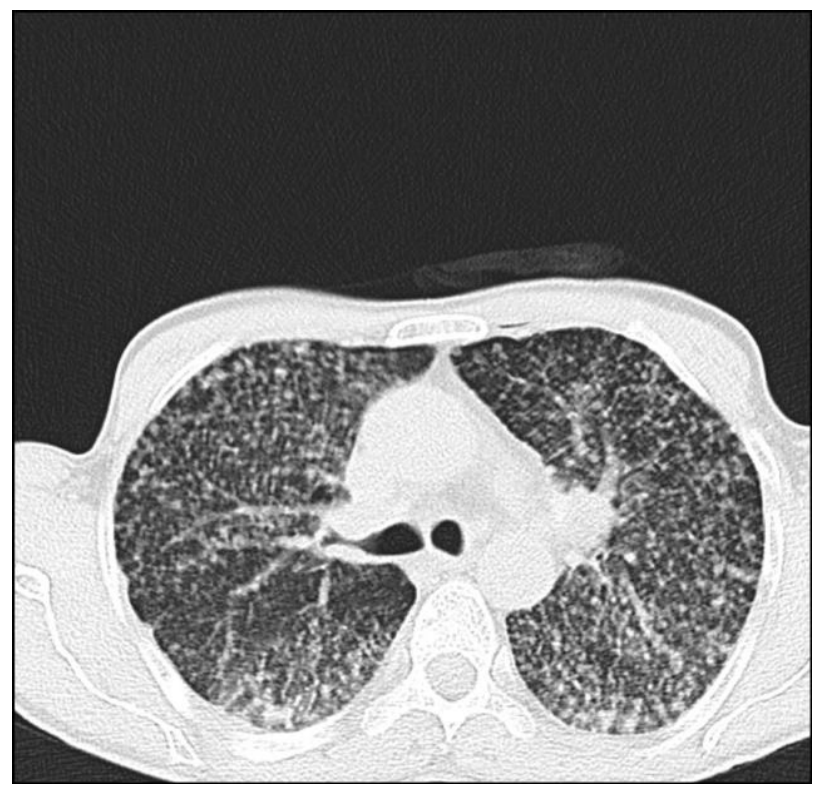

Fig. 2. Small, mildly enhancing, nodular lesion containing central density involving the posterior basal segment of the left lower lobe with a few enlarged pretracheal, retrocaval, aortopulmonary, and right hilar lymph nodes

material (Figure 5), immunohistochemical TTF-1 (Thyroid Transcription Factor -1 ) + nuclear staining to support that the primary localization of this image is the lung (Figure 6).

At the result of PET/CT (Positron Emission Tomography), the pathological increased FDG uptake in miliary style nodules (SUVmax 7.9), left suboccipital, left superior jugular, posterior cervical

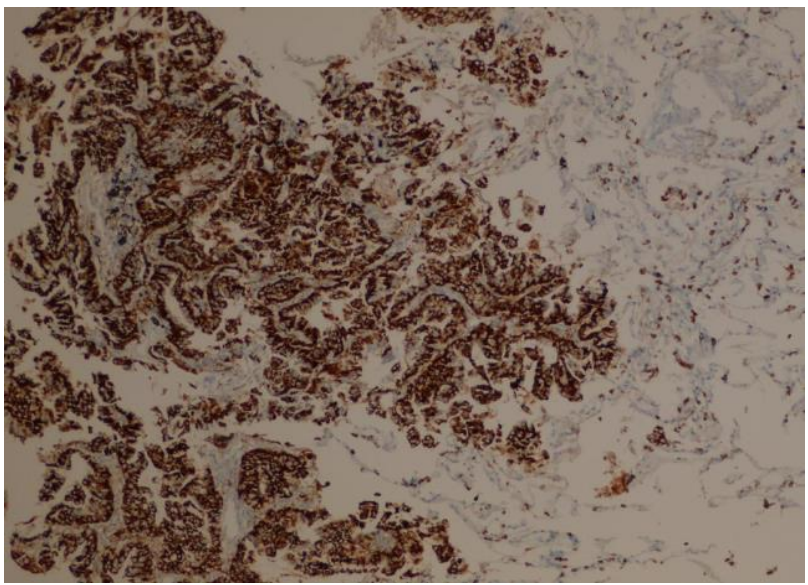

Fig. 3. Primary lung adenocarcinoma with multiple foci suggestive of a metastatic adenocarcinoma

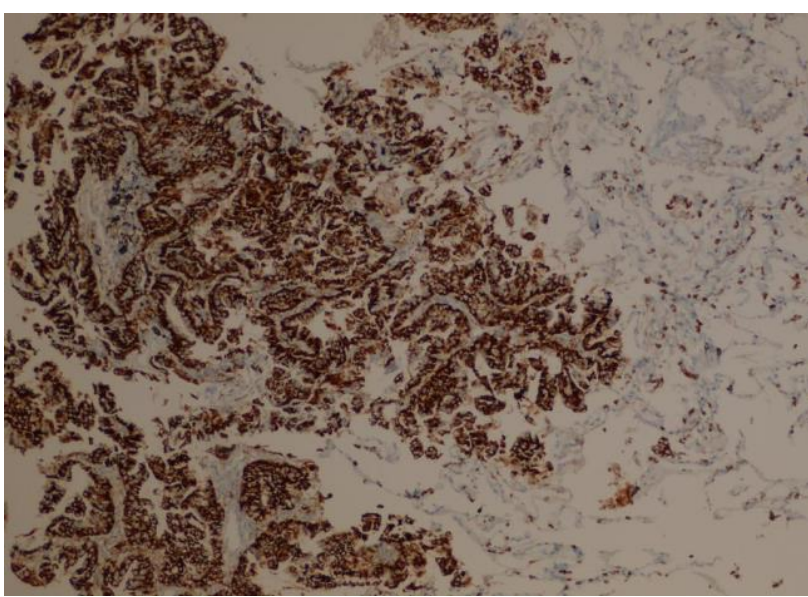

Fig. 4. Immunohistochemical Napsin $\mathrm{A}(+)$ supporting the primary focus of cancer is the lung

and supraclavicular lymphadenopaty (SUVmax 19.2), sclerotic lesions in vertebral colon, pelvic bones (SUVmax12.3). The patient was referred to oncology for chemotherapy.

\section{Discussion}

Imaging findings of lung metastases may be pulmonary nodule, pleural effusion and lymph node enlargement (6). However, the scattered distribution of lung cancers is very rare. The reticulonoduler and miliary distribution is not specific clinical situation but can be seen in many diseases. Tuberculosis is the first diagnosis that comes in mind in developing countries as in our country (7). However there was no anamnesis and clinical findings to support tuberculosis.

Miliary infectionas, interstitial lung diseases and hematogenous metastases show random distribution in the secondary lobes of the lung $(8,9)$. These diagnosis can not be distinguished by CT imaging alone. By the way in our patient bronchoscopy results were negative. 


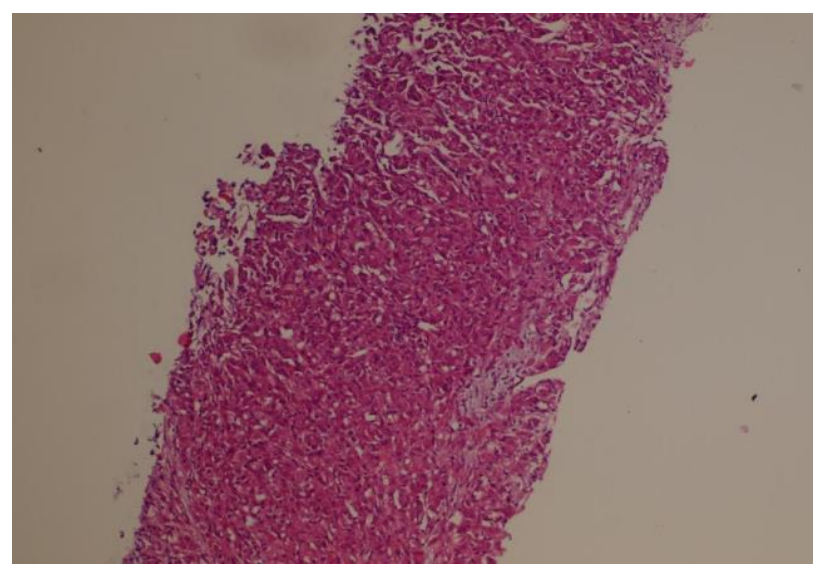

Fig. 5. Adenocarcinoma metastasis in lymph node biopsy material

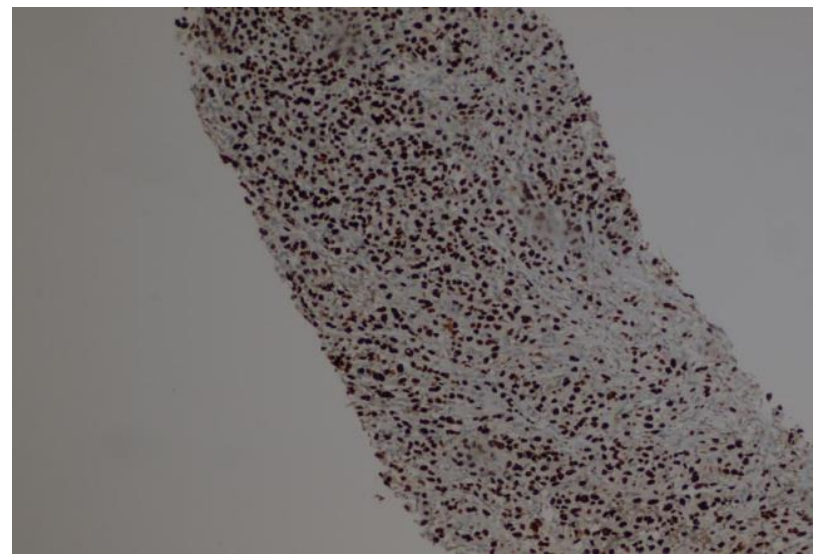

Fig. 6. Immunohistochemical TTF-1 (Thyroid Transcription Factor -1) + nuclear staining to support that the primary localization of this image is the lung

For this reason, we planned a tru-cut biopsy considering common parenchymal involvement, which was partially less invasive and ultimately we were able to diagnose primary lung invasive adenocarcinoma. Tru-cut biopsy sensitivity increases to $75-97 \%$ in benign lesions and $88-95 \%$ in malignant lesions (10). The incidence of complications such as pneumothorax, haemotorax, hemoptysis is lower than surgical techniques (11).

The classification of adenocarcinoma is classified under three main headings as preinvasive lesions, invasive adenocarcinoma and invasive adenocarcinoma variants. The diagnosis was previously described as bronchoalveolar adenocarcinoma but this subclass was changed. It is divided into more specific sub groups as adenocarcinoma insitu, minimally invasive adenocarcinoma, lepidic predominant adenocarcinoma, invasive mucinous adenocarcinoma (12).

When evaluating miliary shadows, primary lung cancer with a hematogenous spread must be in our preliminary diagnosis. Although nonspecific, radiographic finding may be helpful in that cases. However, tissue diagnosis play an important role in establishing the definitive diagnosis. Tru-cut biopsy should be kept in mind as a less invasive method to go to the diagnosis.

\section{References}

1. Chandrasekhar HR, Shashikala P, Murthy BN, Vidyasagar B, Rao HL. Bronchioloalveolar carcinoma mimicking miliary tuberculosis. J Assoc Physicians India 2001; 49: 281-282.

2. Zompatori M, Bnà C, Poletti V, Spaggiari E, Ormitti F, Calabrò E, et al. Diagnostic imaging of diffuse infiltrative disease of the Lung. Respiration 2004; 71: 4-19.

3. Pillai S, Khan A, Khan S, Adenocarsinoma of the Lung Presenting with Intrapulmonary Miliary Metastasis. Cureus 2019; 11: e5430.

4. Furqan M, Butler J. Miliary pattern on chest radiography: TB or not TB? Mayo Clin Proc 2010; 85: 108.

5. Uzer F, Coskun HS, Cilli A. Adenocarcinoma Presenting With Miliary Intrapulmonary Carcinoma Respir Case Rep 2017; 6: 114-117.

6. Quinn D, Gianlupi A, Broste S. The changing radiographic presentation of bronchogenic carcinoma with reference to cell types. Chest 1996; 110: 1474-1479.

7. Sharma BB. Miliary nodules on chest radiographs: A diagnostic dilemma. Lung India 2015; 32: 518520.

8. Voloudaki AE, Tritou IN, Magkanas EG, Chalkiadakis GE, Siafakas NM, Gourtsoyiannis N. HRCT in miliary lung disease. Acta Radiol 1999; 40: 451-456.

9. Raoof S, Amchentsev A, Vlahos I, Goud A, Naidich DP. Pictorial essay: multinodular disease. a high-resolution CT scan diagnostic algorithm. Chest 2006; 129: 805-815.

10. Hiraki $\mathrm{T}$, Mimura $\mathrm{H}$, Gobara $\mathrm{H}$, et al. CT Fluoroscopy-Guided Biopsy of 1,000 Pulmonary Lesions Performed With 20-Gauge Coaxial Cutting Needles: Diagnostic Yield and Risk Factors for Diagnostic Failure. doi: 10.1378/chest.09-0370.

11. Shaham D. Semi-invasive and invasive procedures for the diagnosis and staging of lung cancer. I. Percutaneous transthoracic needle biopsy Radiol Clin North Am 2000; 38: 525-534.

12. Russell PA, Wainer Z, Wright GM, Daniels M, Conron M, Williams RA. Does lung adenocarcinoma subtype predict patient survival? A clinicopathologic study based on the new International Association for the Study of Lung Cancer / American Thoracic Society / European Respiratory Society. International multidisciplinary lung adenocarcinoma classification. J Thorac Oncol 2011; 6: 1496-1504. 